\title{
Studies of physicochemical properties of graphite oxide and thermally exfoliated/reduced graphene oxide
}

\author{
Sabina Elżbieta Drewniak"1, Tadeusz Piotr Pustelny', Roksana Muzyka², Agnieszka Plis ${ }^{2}$ \\ ${ }^{1}$ Silesian University of Technology, Krzywoustego 2, 44-100 Gliwice, Poland \\ ${ }^{2}$ Institute for Chemical Processing of Coal, Zamkowa 1, 41-803 Zabrze, Poland \\ "Corresponding author: e-mail: Sabina.Drewniak@polsl.pl
}

\begin{abstract}
The aim of the experimental research studies was to determine some electrical properties of graphite oxide and thermally exfoliated/reduced graphene oxide. The authors tried to interpret the obtained physicochemical results. For that purpose, both resistance measurements and investigation studies were carried out in order to characterize the samples. The resistance was measured at various temperatures in the course of composition changes of gas atmospheres (which surround the samples). The studies were also supported by such methods as: scanning electron microscopy (SEM), Raman spectroscopy (RS), atomic force microscopy (AFM) and thermogravimetry (TG). Moreover, during the experiments also the elemental analyses (EA) of the tested samples (graphite oxide and thermally exfoliated/reduced graphene oxide) were performed.
\end{abstract}

Keywords: graphite oxide, thermally exfoliated/reduced graphene oxide, topography investigations, Raman spectroscopy, elemental analysis.

\section{INTRODUCTION}

The history involving the fabrication of graphite oxide dates back more than 150 years ago when B. C Brodie prepared graphite oxide for the first time. For that purpose he treated graphite with nitric acid and potassium chlorate ${ }^{1}$. Nowadays, the methods developed by Hummer, Staudenmaier ${ }^{2}$, Hoffmann ${ }^{3}$ and obviously B. C Brodie are basic preparation methods of graphite oxide. Scientists in many research centers have been working on the fabrication of materials by oxidizing graphite and its further reduction", ${ }^{4,5}$. Researchers from the "Institute of Electronic Materials Technology - ITME" in Warsaw (Poland) and from "Grupa Azoty", Tarnow (Poland) are trying to improve the production methods of graphene from the chemically reduced graphene oxide. Moreover, they started research studies aiming at the exfoliation of graphite, using organic solvents. Both methods are used in the production of highly defected graphene flake, suitable for application in the areas of optics and medicine, as well as in the automotive, aircraft and chemical industries ${ }^{6}$.

Currently, many research studies are focused on the application of graphite oxide and on the materials obtained by its modification (e.g. thermally reduced graphene oxide). Expanded graphene oxide has been applied as molecular membranes ${ }^{7}$ and also for the production of hydrogels (which can be used in biotechnology ${ }^{8}$ ). One of the most important branches of industry which applies materials obtained by the modification of graphite is sensor technology ${ }^{9}$, especially gas sensor technology ${ }^{10-12}$. The materials obtained by the modification of graphite seem to be as good as other (commonly applied) materials like $\mathrm{TiO}_{2}, \mathrm{ZnO}^{13}$, graphene ${ }^{14}$ or carbon nanotubes ${ }^{15-16}$.

Basing on their own experience ${ }^{4,11,17-18}$, the authors observed that various acquisition methods of graphite oxide and their modifications (e.g. thermally exfoliated/ reduced graphene oxide) affect their properties. It should be noted that further treatment affects the properties of the analyzed materials. Graphite oxide displays very poor electrical properties, so the modifications are necessary to dissect and remove oxygen from the structure ${ }^{\mathbf{1 9}}$. It has been observed that a longer heat treatment of graphite oxide increases its thermal and electrical conductivity ${ }^{20}$. Therefore, it seems to be reasonable to carry out basic research studies in order to get to know and to understand the properties of carbon-based materials. The authors of this paper are working on the properties of graphite oxide and exfoliated/reduced graphene oxide in terms of their application as a sensitive layer in sensor systems.

\section{EXPERIMENTAL}

\section{Preparation of the samples}

The basic material in our experiments was graphite oxide and thermally exfoliated/reduced graphene oxide. The graphite oxide was prepared with the application of the modified Hummer's method ${ }^{21}$. In that process, commercial natural graphite powder $(90 \mu \mathrm{m})$, supplied by Graphit Kropfmühl AG $\left(\mathrm{L}_{\mathrm{a}}-58 \mathrm{~nm}, \mathrm{~L}_{\mathrm{c}}-29 \mathrm{~nm}\right.$, $\mathrm{d}_{002}-0.338 \mathrm{~nm}$ and $\left.\mathrm{C}^{\text {daf }} 99.5 \%\right)$ was used. Concentrated $\mathrm{H}_{2} \mathrm{SO}_{4}(95-97 \%)$ was used as an acid and $\mathrm{KMnO}_{4}$ and $\mathrm{NaNO}_{3}$ as oxidants. A graphite sample $(1 \mathrm{~g})$ with a < $20 \mu \mathrm{m}$ particle size (ground in a planetary ball mill, PM100) was mixed with the acid and placed in a bath of water and ice. The oxidizing agent was partially added in the course of continuous mixing until a homogeneous paste had been achieved. The reaction temperature was within the range $5 \div 25^{\circ} \mathrm{C}$. In such a method, the following amounts of reagents were used: $30 \mathrm{ml}$ of $\mathrm{H}_{2} \mathrm{SO}_{4}$, $3 \mathrm{~g} \mathrm{NaNO}_{3}$. After 2-hour mixing, oxidized products were obtained. In the next step, the obtained oxidized mixtures were diluted in $100 \mathrm{ml}$ of mili-Q water and 3\% $\mathrm{H}_{2} \mathrm{O}_{2}$ was slowly added. As the next step, the mixture was stirred for $30 \mathrm{~min}$ and then centrifuged $(5000 \mathrm{rpm}$, $15 \mathrm{~min}$ ). As the next step, the supernatant began to decant away and the received solid material was washed with water mixture and centrifuged again. Such a process was repeated until $\mathrm{pH}$ became neutral. Finally, the graphite oxide was dried overnight under vacuum at $50^{\circ} \mathrm{C}$ and stored in the presence of $\mathrm{P}_{2} \mathrm{O}_{5}$ as desiccant. 
Thermally exfoliated/reduced graphene oxide (further on referred to as reduced graphene oxide) was the second material used in our experiments. The reduced graphene oxide was prepared from graphite oxide (the preparation of which has been described above). The graphite oxide was reduced by annealing under a nitrogen atmosphere $(5 \mathrm{~min})$ at a temperature of $900^{\circ} \mathrm{C}$.

Both graphite oxide and the reduced graphene oxide were imposed on the resistive structure in the same way. The sensing layers (graphite oxide and reduced graphene oxide) were mixed with a small amount of anhydrous ethanol and then applied onto the structure and left for 48 hours to dry.

\section{Characterization of the structures}

All the experiments with the application of graphite oxide and the reduced graphene oxide were performed in parallel and will be described in the following sections of this work.

The qualitative and quantitative composition of the selected elements was determined using an elemental analyser, viz. Vario Macro Cube - Elementar Analysensysteme GmbH. Such equipment allows us to determine the content of oxygen, carbon, hydrogen, sulphur and nitrogen.

The differences in topography were confirmed by measurements using scanning electron microscopy (SEM) and atomic force microscopy (AFM). The SEM images were obtained using the Inspect S50 (FEI) system (measurement parameters: $\mathrm{HV}: 2.0 \mathrm{kV}$, spot: 2.0 , detector type: ETD).

The AFM measurements were carried out in compliance with the NTEGRA Prima system (the NTMDT company) with HA_NC tip. The images were obtained using the intermittent contact mode (the resonant frequency was $255.965 \mathrm{kHz}$ for the measurement of graphite oxide and $255.986 \mathrm{kHz}$ for the measurement of the thermally exfoliated/reduced graphene oxide).

Raman spectra were obtained using the N-TEGRA Spectra system (NT-MDT). The wavelength of the used laser was equal to $532 \mathrm{~nm}$.

Thermogravimetric (TG) and differential TG (DTG) curves were obtained using a Netsch TGSTA209LUXX. The samples were tested within the range from 40 to $1000^{\circ} \mathrm{C}$, with a heating rate of $10 \mathrm{~K} \mathrm{~min}^{-1}$ under an argon flow $25 \mathrm{ml} \mathrm{min}^{-1}$.

In order to measure the resistance of the sample, the structures were placed in the measuring chamber (a strictly controlled gas atmosphere was dosed to that chamber). All structures were connected in a parallel way to eliminate any differences of the temperature of the substrate, the dosed gas atmosphere or the composition of the gas. The resistance was measured in regular time intervals, enabling the determination of the difference between the resistance during the dosing of the synthetic air and the stabilized resistance during the dosing of hydrogen with the concentration of $4 \%$ in the synthetic air.

\section{RESULTS AND DISCUSSION}

Table 1 shows the qualitative and quantitative composition of the selected elements. The obtained results show that all the analyzed materials consisted principally of carbon. Graphite contains trace amounts of other elements. Graphite oxide is rich in additional functional groups, which consist mainly of oxygen and hydrogen. It is possible that such additional groups also contain carbon (eg. carbonyl groups). In graphite oxide the amount of other elements (sulphur and nitrogen) is very low. After the reduction process, the percentage content of carbon is higher than that in the case of graphite oxide. It may be concluded that the percentage contents of hydrogen, sulphur and oxygen were decreased It means that the additional groups must be released from the analyzed materials. The content of nitrogen was still very low.

Such significant changes in the content of the respective elements suggest that graphite oxide and reduced graphene oxide display a different topography and different chemical and physical properties. Figure 1 shows

Table 1. Content of carbon, hydrogen, nitrogen, sulphur and oxygen in analyzed materials ( $*$ the contents of individual elements for graphite was converted to a dafstate, which is understood as anhydrous and ash-free)

\begin{tabular}{|l|c|c|c|}
\hline \multirow{2}{*}{ Component } & \multicolumn{3}{|c|}{ Tested material } \\
\cline { 2 - 4 } & Graphite, $\left.{ }^{*} \%\right]$ & Graphite oxide, [\%] & $\begin{array}{c}\text { Thermally exfoliated/reduced } \\
\text { graphene oxide, [\%] }\end{array}$ \\
\hline $\mathrm{C}$ & 99.50 & 63.80 & 89.80 \\
\hline $\mathrm{H}$ & 0.09 & 2.17 & 0.44 \\
\hline $\mathrm{N}$ & 0.20 & 0.08 & 0.26 \\
\hline $\mathrm{S}$ & 0.05 & 1.62 & 0.95 \\
\hline $\mathrm{O}$ & 0.16 & 32.29 & 8.53 \\
\hline
\end{tabular}
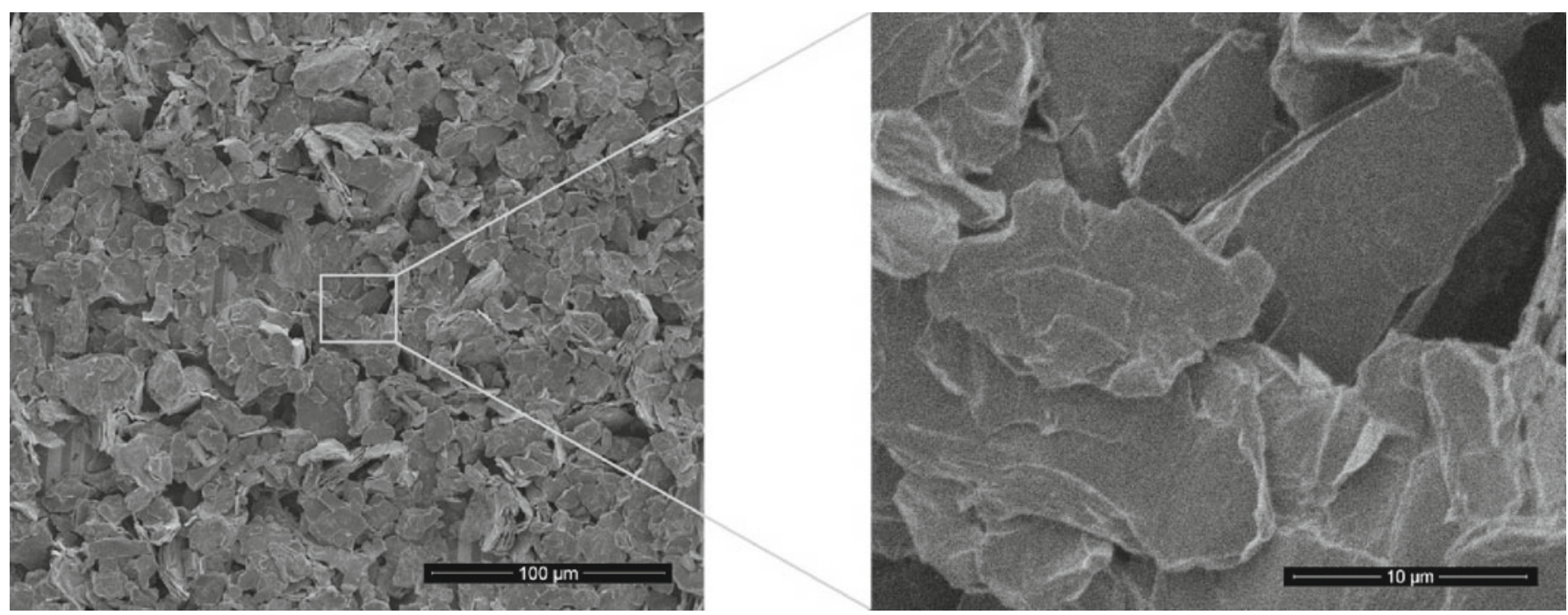

Figure 1. SEM image of graphite oxide 
the topography of the analyzed graphite oxide sample. The surface of this material is not homogenous - the grains differ in size (there are grains within the range from a few to 40 micrometers). The size of the gains of the reduced graphene oxide is more homogeneous, averaging approximately from 10 to $20 \mu \mathrm{m}$. Figure 2 shows the topography of the thermally exfoliated/reduced graphene oxide. The surface of the reduced graphene oxide is significantly better developed - the layers of that material were delaminated, forming a porous structure. Moreover, it can be noticed that the surface of the grains of graphite oxide is relatively smoother in comparison with the surface of the grains of the reduced graphene oxide. The topography of graphite oxide can be also seen in Figure 3 and the topography of the thermally reduced/ exfoliated graphene oxide in Figure 4. Such images were obtained by means of atomic force microscopy (AFM).
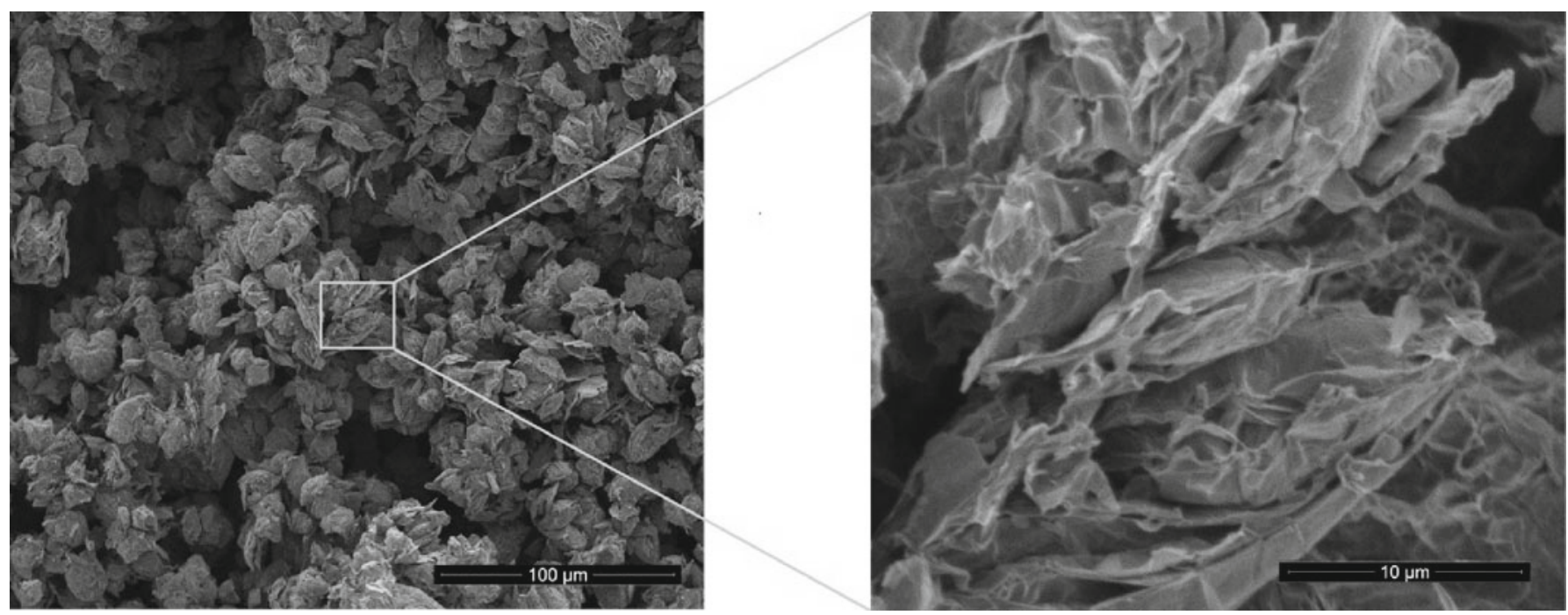

Figure 2. SEM image of thermally exfoliated/reduced graphene oxide
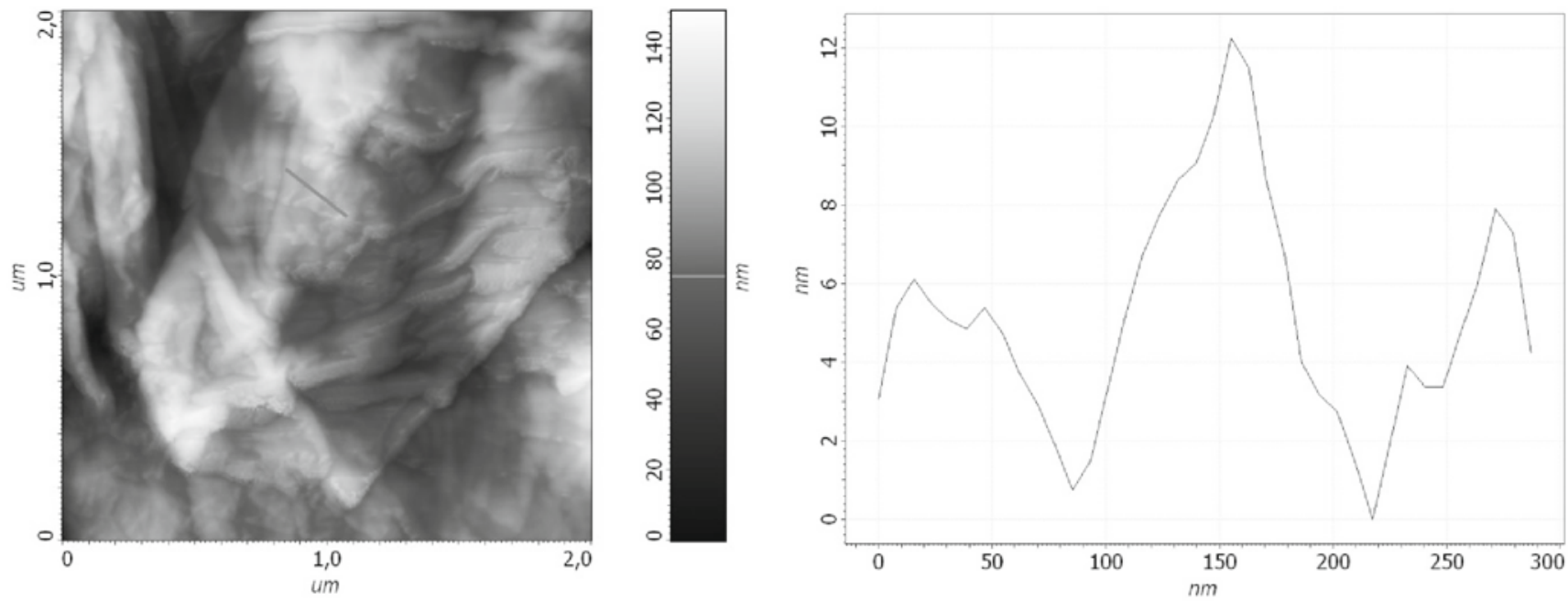

Figure 3. AFM image of graphite oxide
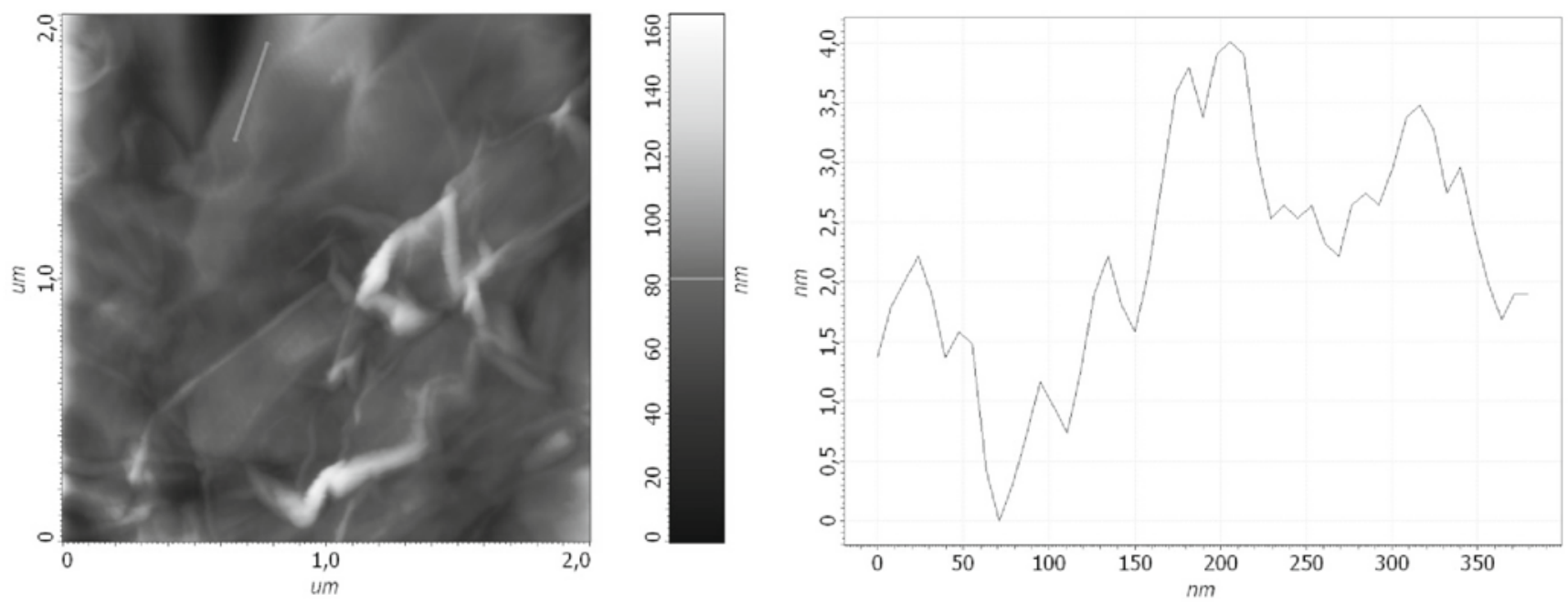

Figure 4. AFM image of thermally exfoliated/reduced graphene 
The obtained measurement results using the Raman spectroscopy (RS) confirm the hypothesis that the structure of the reduced graphene oxide was delaminated. A typical Raman spectrum of graphite oxide and reduced graphene oxide is characterized by two characteristic peaks: $1300 \mathrm{~cm}^{-1}$ (peak "D") and $1580 \mathrm{~cm}^{-1}$ (peak “G”). The first one („D”) originates from the breathing modes of six-membered rings that are activated by defects. The second one ("G") is due to the $\mathrm{E}_{2 \mathrm{~g}}$ phonon in the Brillouin zone center ${ }^{22}$. The differences in defects (degree) can be determined by analyzing the value of the aforementioned peaks. The results of the calculations $\left(\mathrm{I}_{\mathrm{D}} / \mathrm{I}_{\mathrm{G}}\right)$ for graphite oxide and thermally exfoliated/reduced graphene oxide are equal to 1.09 and 1.13, respectively. Exemplary spectra are shown in Figure 5. The received differences prove that the number of defects in the structure increased considerably after the reduction process. This may be caused by the disconnection of a part of the functional groups and/or by the damage of the existing crystallographic structure.

The fact that the additional functional groups are removed from the analyzed structure can be confirmed by the thermogravimetric technique (TG). Figure 6 presents the weight loss of graphite oxide as a function of temperature (blue line) and the weight loss of graphite oxide per minute, presented as a function of temperature (red line). It can be seen that the most significant changes are taking place at a temperature of $\sim 200^{\circ} \mathrm{C}$ - above this temperature, the changes in weight of this material are taking place to a limited extent, due to the fact that the residual functional groups (hydroxyl $-\mathrm{OH}$, epoxy $-\mathrm{C}-\mathrm{O}-\mathrm{C}$ and carbonyl $-\mathrm{C}=\mathrm{O}$ ) are still attached

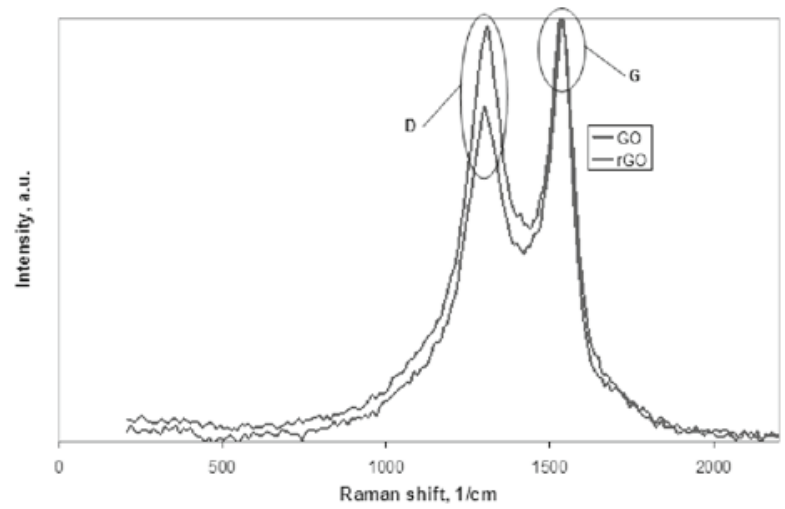

Figure 5. Raman spectra of graphite oxide and reduced graphene oxide

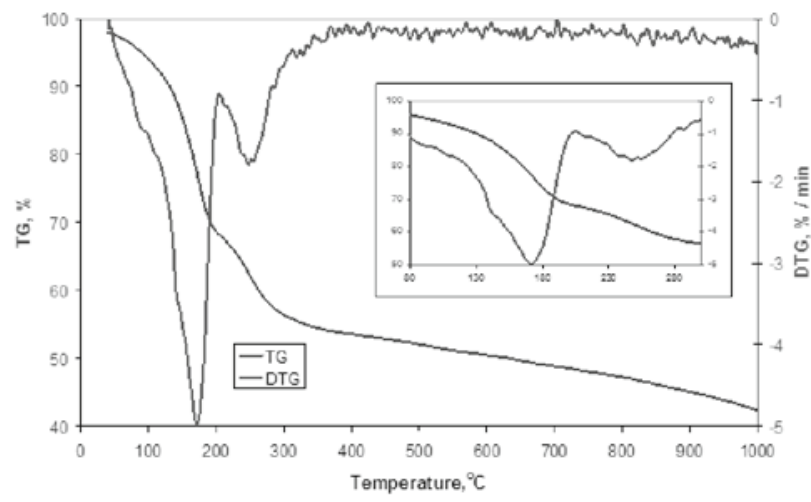

Figure 6. Weight loss as a function of temperature to the surface despite thermal exfoliation/reduction of graphite oxide.

Analyzing the information quoted above, it can be seen that the pyrolysis occurs in several steps. In the first step (from $40^{\circ} \mathrm{C}$ to $115^{\circ} \mathrm{C}$ ), the maximum DTG temperature amounts to $880^{\circ} \mathrm{C}$ (the change in the weight is then equal to $4.0 \%$ ) The weight change is (in this case) associated with the loss of moisture from the samples. The second pyrolysis step occurs within the range of temperature from $115^{\circ} \mathrm{C}$ to $205^{\circ} \mathrm{C}$ and from $205^{\circ} \mathrm{C}$ to $1000^{\circ} \mathrm{C}$. In the temperature range from $115^{\circ} \mathrm{C}$ to $205^{\circ} \mathrm{C}$, the maximum DTG temperature is equal to $172^{\circ} \mathrm{C}$ with a loss of weight: $25.9 \%$, while in the temperature range from $205^{\circ} \mathrm{C}$ to $1000^{\circ} \mathrm{C}$, the maximum DTG temperature amounts to $246^{\circ} \mathrm{C}$ with a loss of weight: $25.7 \%$. The second step of pyrolysis is associated with the disconnection of the functional groups from the surface (from the oxidized graphene planes) ${ }^{\mathbf{2 3}}$.

The SEM image of the resistance structure with the reduced graphene oxide was presented in Figure 7. Figure 8 presents the differences between the resistances in the above mentioned gas atmospheres as a function of temperature for the structure with graphite oxide. Similar characteristics are shown in Figure 9 (for the structure

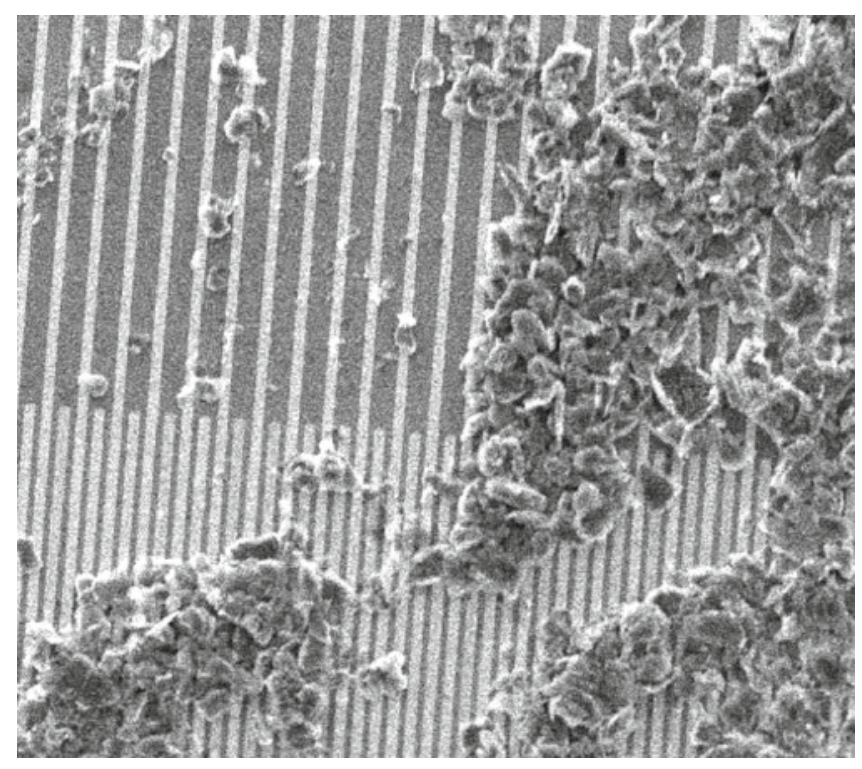

Figure 7. SEM image of the resistance structure with thermally exfoliated/reduced graphene oxide nanoelements

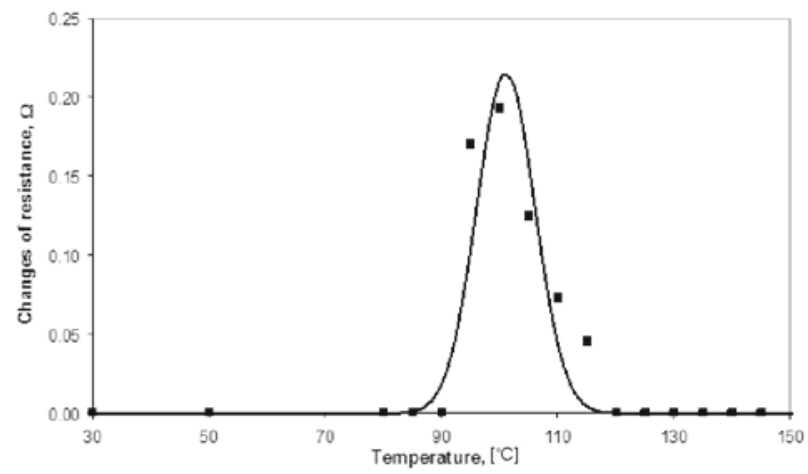

Figure 8. Changes of resistance of structure with graphite oxide resulting from changes in surrounding atmosphere (synthetic air/4\% hydrogen in synthetic air) - the first attempt 
with the reduced graphene oxide). It should be noted that Fig. 8 and Fig. 9 present the results of the previous experiment. In the case of both structures, the optimal temperature for which the changes of resistance reach their maximum can be determined. In the structure with graphite oxide, the optimal temperature was $\sim 100^{\circ} \mathrm{C}$, while in the structure with the reduced graphene oxide, the optimal temperature was $\sim 130^{\circ} \mathrm{C}$. None of the structures showed any sensitivity (the resistances did

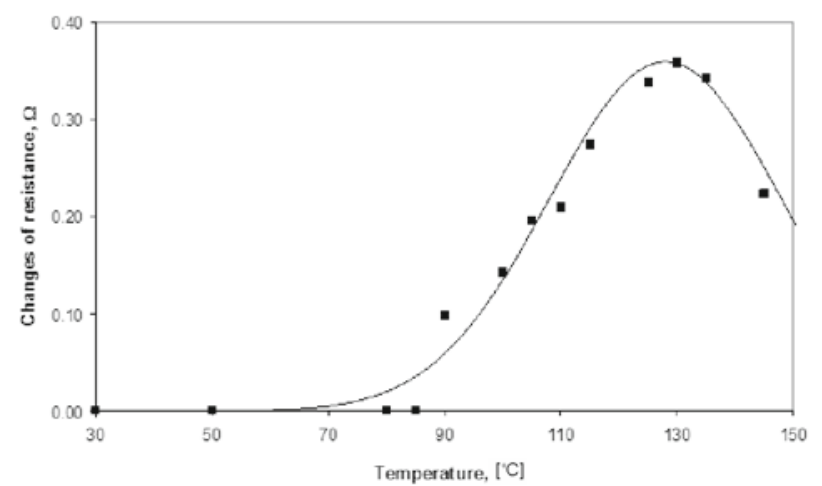

Figure 9. Changes of resistance of structure with thermally exfoliated/reduced graphene oxide resulting from changes in surrounding atmosphere (synthetic air/4\% hydrogen in synthetic air) - the first attempt

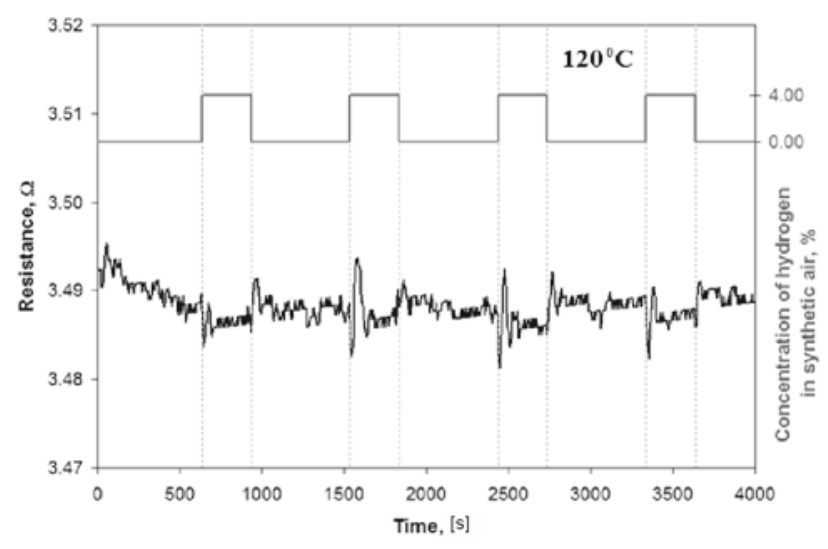

Figure 10. Changes of resistance of the structure with graphite oxide as a function of time during dosing the various gas atmospheres (synthetic air/4\% hydrogen in synthetic air)

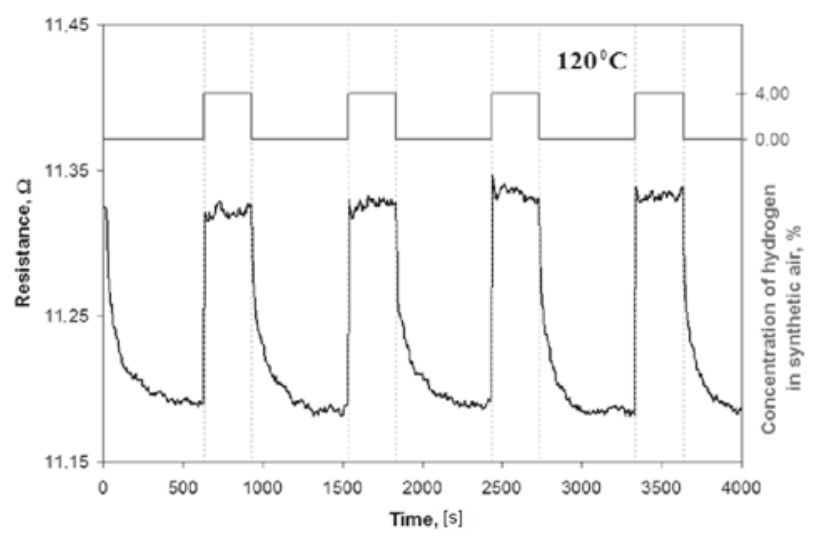

Figure 11. The changes of resistance of the structure with reduced graphene oxide as a function of time during dosing the various gas atmospheres (synthetic air $/ 4 \%$ hydrogen in synthetic air) not change during the changes in the composition of the dosing atmosphere) below $70^{\circ} \mathrm{C}$. Moreover, it can be seen that the structure with graphite oxide did not react also above $120^{\circ} \mathrm{C}$.

Further on, a few series of similar measurements were performed in order to check the stability of the structures. Unfortunately, the structure with graphite oxide stops reacting to the changes of the composition in the dosing atmosphere after its first annealing (Fig. 10). The structure with the reduced graphene oxide still reacts distinctly (Fig. 11).

Fig. 12 shows the analogous characteristics to those shown in Fig. 9 (the structure with the reduced graphene oxide as a sensitive layer). The measurements were carried out several months after the first experiment. It can be seen that the maximum changes of resistance take place in the range of temperature between $140^{\circ} \mathrm{C}$ and $160^{\circ} \mathrm{C}$, which is slightly larger as compared to the one determined during the first experiment. The changes of the resistance are also smaller than the changes determined after the first experiment. The described behavior may have been caused by the fact that in the previous test the structures were very sensitive to temperature. It should be noted that the sensitive layer may contain trace amounts of ethanol. After the first annealing (first experiment), the additional functional groups, originating from ethanol, are removed from the sensitive layer. The absence of ethanol may also cause a lack of sensitivity of the structure of graphite oxide in subsequent experimental trials. In that case, the sensing layer of graphite oxide was not modified enough ( too low temperature of annealing, different composition of the environment) to obtain a layer which is similar (with similar electrical properties) to the layer of the thermally exfoliated / reduced graphene oxide. Lower sensitivity of the sample with reduced graphene oxide, which was observed in further experiments, may have also been caused by the absence of ethanol. Moreover, it can be concluded that the delamination of the surface also affects the sensitivity (larger area of interaction).

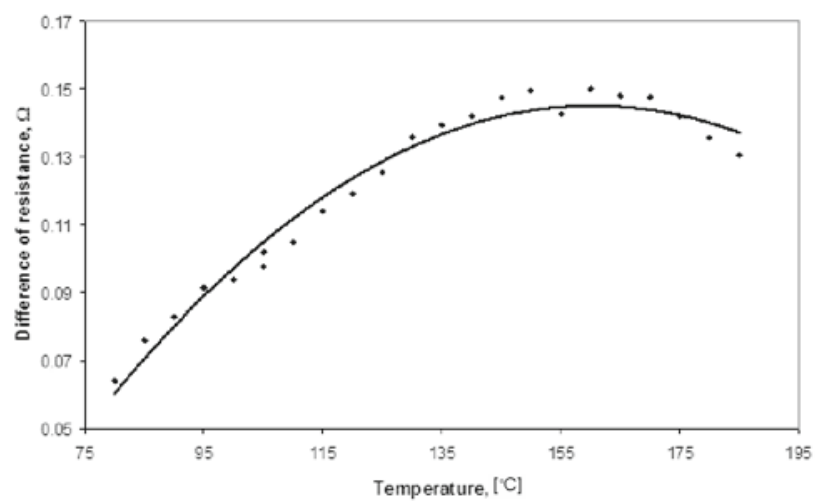

Figure 12. Changes of resistance of structure with thermally exfoliated/reduced graphene oxide resulting from changes in surrounding atmosphere (synthetic air/4\% hydrogen in synthetic air) - the another attempt 


\section{CONCLUSIONS}

The investigation studies allowed us to get a better knowledge of physical and chemical properties of materials based on graphite oxide. The presented studies suggest that after the reduction and exfoliation process, the structures are characterized by stable properties. In such structures a part of the functional groups (which were present in graphite oxide) was removed. Consequently, the remaining bonds of thermally exfoliated/ reduced graphene oxide can interact with the surrounding atmosphere. Thermally exfoliated/reduced graphene oxide can be used as a sensing layer for the detection of selected gases. Our current investigation studies show that the reduced graphene oxide is also sensitive to other gaseous environments. Moreover, it is also sensitive to the changes of temperature. Our future research studies will be focused on the examination of the sensitivity of the reduced graphene oxide to other gases (eg. $\mathrm{NO}_{2}$, $\mathrm{CO}, \mathrm{CO}_{2}, \mathrm{NH}_{3}, \mathrm{CH}_{4}$, alcohol vapor and water vapor).

\section{ACKNOWLEDGMENT}

The work was partially sponsored by:

- the Polish National Science Centre (NCN) within

the grant 2012/07/B/ST7/01 471;

- the Statutory R\&D Project IChPW, no 11.15.024.

\section{LITERATURE CITED}

1. Eda, G. \& Chhowalla, M. (2010). Chemically derived graphene oxide: towards large-area thin-film electronics and optoelectronics. Adv. Mater. 22(22), 2392-2415. DOI: 10.1002/adma.200903689.

2. Stankovich, S., Dikin, D.A., Piner, R.D.,. Kohlhaas, K.A., Kleinhammes, A., Jia, Y., Wu, Y., Nguyen, S.T. \& Ruoff, R.S. (2007). Synthesis of graphene-based nanosheets via chemical reduction of exfoliated graphite oxide. Carbon 45(7), 1558-1565. DOI:10.1016/j.carbon.2007.02.034.

3. Wong, C., Jankovsky, O., Sofer, Z. \& Pumera, M. (2014). Vacuum-assisted microwave reduction/exfoliation of graphite oxide and the influence of precursor graphite oxide. Carbon 77, 508-517. DOI: 10.1016/j.carbon.2014.05.056.

4. Drewniak, S., Pustelny, T., Muzyka, R., Konieczny, G. \& Kałużyński, P. (2014). The effect of oxidation and reduction processes on physicochemical properties of graphite oxide and reduced graphene. Photo. Lett. Pol. 6(4) 130-132. DOI: 10.4302/plp.2014.4.06.

5. McAllister, M., Li, J., Adamson, D., Schniepp, A.A., Liu, J., Herrera-Alonso, M., Milius, D., Car, R., Prud'homme, R. \& Aksay, A. (2007). Single Sheet Functionalized Graphene by Oxidation and Thermal Expansion of Graphite., Chem. Mater. 19(18), 4396-4404. DOI: 10.1021/cm0630800.

6. Lipińska, L., Koziński, R., Jagiełło J., Librant, K., Aksienionek, M. \& Wiliński, Z. (2012). Chemical methods of obtaining graphene flakes. Chem. Przem. 5, 16-19. (In Polish).

7. Pacile, D., Meyyer, J., Rodriguez, A., Papagno, M., GomezNavarro, C., Sundaram, R., Burghard, M., Kern, K., Carbone, C. \& Kaiser, U. (2011). Electronic properties and atomic structure of graphene oxide membranes. Carbon 49, 966-972. DOI: 10.1016/j.carbon.2010.09.063.

8. Sheng, K., Xu, Y., Li, C. \& Shi, G. (2011). High-performance self-assembled graphene hydrogels prepared by chemical reduction of graphene oxide. New Carbon Mater. 26(1), 9-15. DOI: 10.1016/S1872-5805(11)60062-0.

9. Schwamb, T., Burg, B.R., Schirmer, N.C. \& Poulikakos, D. (2009). An electrical method for the measurement of the thermal and electrical conductivity of reduced graphene oxide nanostructures. Nanotechnology 20, 405704(5pp). DOI: $10.1088 / 0957-4484 / 20 / 40 / 405704$.

10. Basu, S. \& Bhattacharyya. (2012). Recent developments on graphene and graphene oxide based solid state gas sensors. Sensors and Actuators B: Chemical. 173, 1-21 DOI: 10.1016/j.snb.2012.07.092.

11. Drewniak, S., Pustelny, T., Muzyka, R., Stolarczyk, A. \& Konieczny, G. (2015). Investigations of selected physical properties of graphite oxide and thermally exfoliated/reduced graphene oxide in the aspect of their applications in photonic gas sensors. Photo. Lett. Pol. 7(2), 47-49. DOI: 10.4302/plp.2015.2.06.

12. Hu, N., Yang, Z., Wang, Y., Zhang, L., Wang, Y., Huang, X., Wei, H., Wei, L. \& Zhang, Y. (2014). Ultrafast and sensitive room temperature $\mathrm{NH}_{3}$ gas sensors based on chemically reduced graphene oxide. Nanotechnology 25(2), 1-9. DOI: 10.1088/0957-4484/25/2/025502.

13. Pustelny, T., Procek, M., Maciak, E., Stolarczyk, A., Drewniak, S., Urbanczyk, M., Setkiewicz, M., Gut, K. \& Opilski, Z. (2012). Gas sensors based on nanostructures of semiconductors $\mathrm{ZnO}$ and $\mathrm{TiO}_{2}$. Bull. Pol. Ac.: Tech. 60 (4), 853-859. DOI: 10.2478/v10175-012-0099-1.

14. Pustelny, T., Setkiewicz, M., Drewniak, S., Maciak, E., Stolarczyk, A., Procek, M., Urbanczyk, M., Gut, K., Opilski, Z., Pasternak, I. \& Strupinski, W. (2012). The Influence of Humidity on the Resistance Structures with Graphene Sensor Layer. Acta Phy. Polon. A 122, 870-873. ISSN: 05874246.

15. Kong, J., Franklin, N.R., Zhou, C., Chapline, M.G., Peng, S., Cho, K. \& Dai, H. (2000). Nanotube molecular wires as chemical sensors. Science 287(5453), 622-625. DOI: 10.1126/science.287.5453.622.

16. Dobrzanska-Danikiewicz, A.D., Cichocki, D., Łukowiec, D. \& Wolany, W. (2014). Carbon nanotubes synthesis time versus their layer height. Arch. Mater. Sci. Engine. 69(1), 5-11. ISSN 18972764

17. Pustelny, T., Drewniak, S., Setkiewicz, M., Maciak, E., Urbańczyk, M., Procek, M. Gut, K. Opilski, Z., Jagiello, J. \& Lipinska. L. (2013) The sensitivity of sensor structures with oxide graphene exposed to selected gaseous atmospheres. Bull. Pol. Ac.: Tech. 61(3), 705-710. DOI: 10.2478/ bpasts-2013-0075.

18. Drewniak, S., Pustelny, T., Setkiewicz, M., Maciak, E., Urbańczyk, M., Procek, M., Opilski, Z., Jagiello, J. \& Lipinska, L. (2013). Investigations of SAW Structures with Oxide Graphene Layer to Detection of Selected Gases. Acta Phys. Polon. A 124(3), 402-405. DOI: 10.12693/ APhysPolA.124.402.

19. Wang, S., Geng, Y., Zheng, Q. \& Kim, J. (2010). Fabrication of highly conducting and transparent graphene films. Carbon 48, 1815-1823. DOI: 10.1016/j.carbon.2010.01.027.

20. Dikin, D., Stankovich, S., Zimney, E., Piner, R., Dommett, G., Evmenenko, G. \&Ruoff, R. (2007). Preparation and characterization of graphene oxide paper. Nature 448(7152), 457-460. DOI: 10.1038/nature06016.

21. Hummers, W.S. (1954). U.S. Patent No. 2,798,878. Detroit, Mich.: United States Patent Office.

22. Eigler, S., Dotzer, C. \& Hirsch, A. (2012). Visualization of defect densities in reduced graphene oxide. Carbon 50(10), 3666-3673. DOI: 10.1016/j.carbon.2012.03.039.

23. Zhang, C., Lv, W., Xie, X., Tang, D., Liu, C. \&Yang, Q.H. (2013). Review Towards low temperature thermal exfoliation of graphite oxide for graphene production. Carbon 62, 11-24. DOI: 10.1016/j.carbon.2013.05.033. 